\section{Symptomatic classic convergence insufficiency treated with dot card orthoptic exercise}

\author{
Charles Darko-Takyi, ${ }^{1}$ Kofi Asiedu ${ }^{1,2}$ \\ 'Department of Optometry, University \\ of Cape Coast, Cape Coast; 'Eye Center, \\ Komfo Anokye Teaching Hospital, \\ Kumasi, Ghana
}

\begin{abstract}
Although lots of studies have been reported on convergence insufficiency (CI), no specific case of CI among African patients has been reported. We report a case of symptomatic classic convergence insufficiency in a female student who previously had been misdiagnosed and managed for refractive error. This case highlights the need for comprehensive binocular vision assessment, administration of validated symptom questionnaires in cases of suspicious $\mathrm{CI}$, and the use of simple and inexpensive vision therapy procedures to manage $\mathrm{CI}$ in optometric centres in developing countries.
\end{abstract}

\section{Introduction}

An 18-year-old female student reported to the University of Cape Coast optometric clinic with complaints of intermittent blurred near vision. She disclosed experiencing tearing and some ocular discomforts with near work with all old spectacles prescribed. She reiterated that she was in the middle of her final year exams but her symptoms had worsen lately and she was unable to read for more than twenty-five minutes without experiencing severe visual discomfort. She had not used any topical or systemic medications at the time of consultation. Her medical history was unremarkable. Her family medical and ocular histories were unremarkable.

\section{Case Report}

Visual acuity (VA) was measured in each eye using the Snellen acuity chart and results indicated (Table 1). Versions testing using the broad $\mathrm{H}$ method were done and the results were smooth, accurate, full and extensive in each eye. No movement was detected in unilateral cover test. Amplitude of accommodation was measured using the push-up-to-blur method and the results were $8.50 \mathrm{D}$ in each eye. Near point of convergence (NPC) using Bernell (GR50) accommodation convergence rule with a movable column of 20/30 letters as the target on first visit was receded (Table 1). ${ }^{1}$ The Convergence Insufficiency Symptom Survey (CISS) questionnaire was administered and patient had a high score (Table 1) comparing to a cut off score for her chronological age of less than 16 points. $^{2}$

Pupillary reflex test was done using penlight and the pupils were round, equal and reactive to light in each eye. External eye investigation and internal eye examination using slit lamp biomicroscope and direct ophthalmoscope respectively yielded unremarkable results. Objective and subjective refraction was performed using Keeler streak professional retinoscope and Trial lens set respectively. The distance and near VA through the objective and subjective refraction results (Table 1) with binocular balancing was $6 / 5$ for each eye and N.5 for each eye respectively.

A thorough binocular vision assessment (Table 1) included investigation of the distance and near habitual lateral phorias using von Graefe technique, investigation of positive and negative fusional vergence reserves at near and distance and determination of the gradient accommodative convergence over accommodation (AC/A) ratio. The vertical phoria was orthophoric. The presence of receded NPC of 18/22 cm, high CISS score of 44 points, low AC/A ratio of $1: 1$, low positive fusional reserve of 4/6/1 at near not satisfying Sheard's criteria for a demand of 4 prism diopters of Exophoria per the Convergence Insufficiency Treatment Trial (CITT) model ${ }^{3,4}$ of using the break point lead to a diagnosis of symptomatic classic convergence insufficiency. The patient was educated about her condition and treatment options. The spectacle correction was not prescribed. She consented to undergo vision therapy and hence the dot card (Figure 1) orthoptic exercise was prescribed 5 minutes twice daily for home therapy with close monitoring of weekly telephone calls to ensure compliance.

The dot card is one of the active methods of treatment for $\mathrm{CI}$ and is a training technique in which dots are placed at $3 \mathrm{~cm}$ intervals in a straight line on a paper which is held lengthwise touching the end of the nose so that the dots are 6 to $24 \mathrm{~cm}$ from the eye.,6 This dots serve as targets to train for vergence especially convergence. The exercise was demonstrated to the patient and patient was also made to demonstrate this procedure until it was evident, patient had mastered the art. In this orthoptic procedure, patient kept her head straight and held one end of the dot card against the tip of her nose. She then held the card straight or angled slightly downwards in a position where she could see all the dots properly (Figure 2). Patient then looked at the fur-
Correspondence: Charles Darko-Takyi, Department of Optometry, University of Cape Coast, Cape Coast, Ghana.

Tel. +233.545063571

E-mail: cdarko-takyi@ucc.edu.gh

Key words: Convergence insufficiency; CISS questionnaire; Dot card; Orthoptic exercise.

Contributions: CD-T, took care of the conception and design of this paper, and data acquisition through clinical consultations and follow ups; drafted the manuscript and revised it for important intellectual content; approved the paper for publication and is accountable for all aspects of this work. KA, involved in the data acquisition through clinical consultations, follow up cares and conception and drafting of this paper; revised this paper for important intellectual content and approved the copy for publication.

Conflict of interest: the authors declare no potential conflict of interest.

Consent: written informed consent was obtained from the patient for publication of this Case Report.

Received for publication: 7 October 2015.

Revision received: 13 February 2016.

Accepted for publication: 15 February 2016.

This work is licensed under a Creative Commons Attribution NonCommercial 4.0 License (CC BYNC 4.0).

(C) Copyright C. Darko-Takyi and K. Asiedu, 2016 Licensee PAGEPress, Italy

Optometry Reports 2016; 5:5566

doi:10.4081/optometry.2016.5566

thest dot and tried to make her eye see it as one by gently pulling her eyes inwards. When she saw the dot singly, all the other dots appeared double and they fan outwards towards her. We informed her that this was normal. As she kept the dot single, she made 10 slow counts. As she focused on the further dot accurately and easily, she looked at the next dot up the line closer to her and tried to make it single by gently pulling eyes inwards. When the dots appeared single, all other dots appeared double-making an X pattern crossing through the particular dot she focused on (Figures 2 and 3). This again, we explained to her was normal. She held this position, keeping the dot single, for a short slow count of 10 . She continued in the same way up the line of dots toward her making sure each one is single and holding each one for a count of 10 . If she could not make a dot single, she went back to the previous dot and tried again. Patient was made to understand that sometimes discomfort is felt which is normal and thus she should keep practicing. If however actual pain or headache is felt, patient should relax; if this 
persisted, patient was asked to consult us. The aim of this exercise was to see the dot closest to patient's nose singly whiles holding it for a slow count of 10 . She was instructed that after a month she will return to the clinic for monitoring and follow up care.

She returned after a month and the clinical parameters were measured again. There was an improvement in the following parameters: near VA for right and left eyes, NPC, CISS score and PFV at near (Table 1). Notwithstanding, the NPC value was still receded and CISS score was clinically still very symptomatic. ${ }^{2-4}$ An improvement in positive fusional reserve at near now met Sheard's criteria per habitual phoria of 4 prism diopters of exophoria using the break point as used by the CITT study group. ${ }^{3,4}$ An improvement with the recovery point in the reserve measurement indicated that her vergence system was developing some stamina to say the least about it. She was advised to adhere to the home-based dot card therapy ( 5 minutes of exercise twice daily in morning and evenings, with weekly telephone calls by authors to monitor process) as her symptoms were improving as well as the marginal improvement in the clinical signs.

She returned again after another month for follow up care. All clinical signs measured were greatly improved and CISS score before and after binocular vision assessment was normal for her chronological age (Table 1). ${ }^{2}$ There was an improvement in the recovery point for positive fusional reserve at near indicating further improvement in the stamina of her ver- gence system and also indirectly pointing to improvement in vergence response and facility. She was considered successfully treated since the CISS score treatment endpoint was met ${ }^{2-4}$ her near point of convergence was greatly improved and marginal improvement in near positive fusional reserve was also obtained. Sheard's criteria were met using the break point value. She was advised to continue therapy by doing therapy 15 minutes every week with close monitoring of monthly telephone calls.

\section{Discussion}

CI may occur secondary to systemic diseases, ${ }^{7}$ may be associated with other ocular or neurological disorders ${ }^{8}$ or may present without a known systemic or psychological etiology. ${ }^{9}$ Patients with a high exophoria who have diseases that interfere with normal vision, such as cataracts, may demonstrate a gross convergence insufficiency after cataract surgery. ${ }^{10}$ Symptomatic CI results from the ineffectiveness of the cross-link between accommodation and convergence, voluntary convergence interactions or fusional convergence ${ }^{11}$ which causes a breakdown in binocular vision resulting in the asthenopic symptoms.

Treatment modalities for symptomatic CI includes both passive and active methods. ${ }^{3,4}$ Passive methods involve the use of base-in prisms and spectacle correction especially for near whilst active therapies involve homebased therapy using pencil pushups (HBPP) alone, home-based therapy using pencil pushups plus other therapy techniques, officebased vision therapy/orthoptics. ${ }^{3,4}$ All these methods are aim at normalizing the accommodative and vergence system and their mutual interactions. ${ }^{4}$ It has been shown in clinical trials that office-based vision therapy/orthoptics with home re-enforcement is the most effective treatment for convergence insufficiency. ${ }^{3}$ Notwithstanding, office-based vision therapy/orthoptics may not be practical in certain situations. The dot card is one of the least expensive vision therapy procedures and the only disadvantage is that is difficult to maintain interest in young children as is a paper based design. ${ }^{12}$ Among the simple and less expensive home-based orthoptic exercises, the dot card treat convergence insufficiency more effectively than simple pen push-up exercises by ensuring correct ocular alignment. ${ }^{13}$

Treatment outcomes for this patient included a CISS score of less than 16 points, near point of convergence with break point of $6 \mathrm{~cm}$ or less and positive fusional reserve break point of 15 prism diopters or meeting Sheard's criteria as recommended and used by CITT study group. ${ }^{3}$ The CISS questionnaire has been validated to have the necessary psychometric properties to demonstrate successful treatment $^{2}$ and has been used in clinical trials for such purposes. ${ }^{3,4}$ In the CISS, the Reliable Change Index (RCI) ${ }^{14}$ — a statistic which indicates the number of points crucial to show if a

Table 1. Clinical results for three visits.

\begin{tabular}{|c|c|c|c|}
\hline Test & First visit & Second visit & Third visit \\
\hline Distance visual acuity & $\begin{array}{l}\text { OD } 6 / 5 \\
\text { OS } 6 / 5\end{array}$ & $\begin{array}{l}\text { OS } 6 / 5 \\
\text { OD } 6 / 5\end{array}$ & $\begin{array}{l}\text { OD } 6 / 5 \\
\text { OS } 6 / 5\end{array}$ \\
\hline Near visual acuity & $\begin{array}{l}\text { OD N.6 } \\
\text { OS N.5 }\end{array}$ & $\begin{array}{l}\text { OS N.6 } \\
\text { OD N.5 }\end{array}$ & $\begin{array}{l}\text { OD N.5 } \\
\text { OS N.5 }\end{array}$ \\
\hline $\mathrm{AOA}$ & $\begin{array}{l}\text { OD } 8.50 \mathrm{D} \\
\text { OS } 8.50 \mathrm{D}\end{array}$ & - & - \\
\hline $\mathrm{NPC}(\mathrm{cm})$ & $18 / 22$ & $15 / 17$ & $10 / 11$ \\
\hline CISS score & 44 & 27 & $\begin{array}{l}14 \text { (before testing); } \\
13 \text { (after testing) }\end{array}$ \\
\hline Retinoscopy & $\begin{array}{l}\mathrm{OD}+0.50 \mathrm{DS} /-0.50 \mathrm{DC} \times 180 \\
\mathrm{OS}+0.50 \mathrm{DS} /-0.25 \mathrm{DC} \times 180\end{array}$ & & \\
\hline Subjective refraction & $\begin{array}{c}\mathrm{OD}+0.50 \mathrm{DS} /-0.25 \mathrm{DC} \times 180 \\
\mathrm{OS}+0.25 \mathrm{DS}\end{array}$ & & \\
\hline Distance lateral phoria & Orthophoria & & \\
\hline Near lateral phoria & $4^{\Delta}$ exophoria & & \\
\hline PFV (distance) & $6 / 9 / 3$ & & \\
\hline NFV (distance) & $\mathrm{x} / 6 / 3$ & & \\
\hline PFV (near) & $4 / 6 / 1$ & $6 / 9 / 2$ & $6 / 9 / 3$ \\
\hline NFV (near) & $6 / 9 / 1$ & & \\
\hline AC/A ratio & $1: 1$ & & \\
\hline
\end{tabular}

OD, right eye; OS, left eye; AOA, amplitude of accommodation; NPC, near point of convergence; CISS, convergence insufficiency syptom survey; PVF, positive fusional vergence; NFV, negative fusional vergence; AC/A, accommodative convergence accommodation ratio. 
variation in a score before and after treatment is from true change or is probabilistic- has been used to determine response to treatment and has been estimated to be within-subject

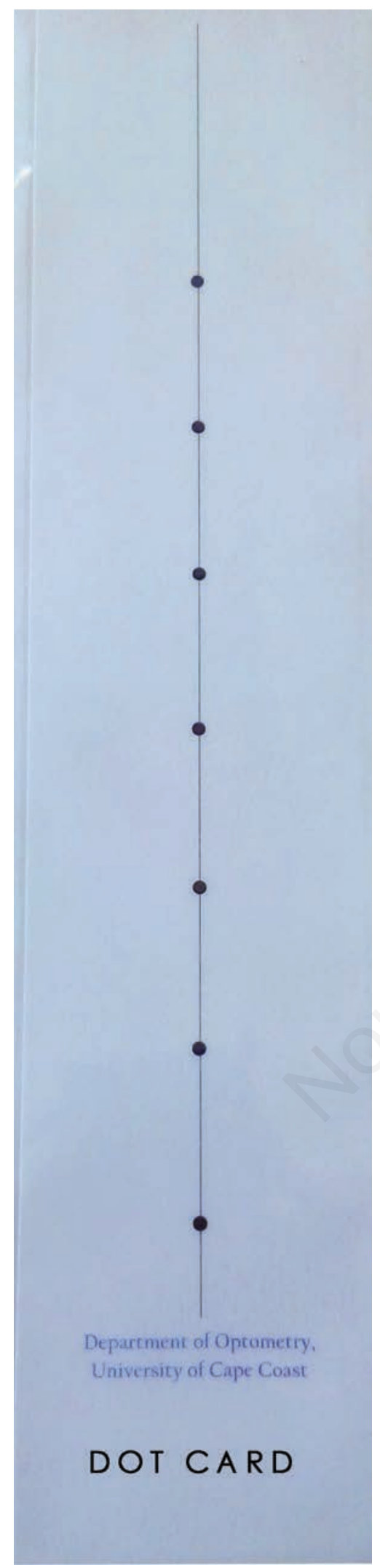

Figure 1. Dot card. change in symptom level of 8.0 points. ${ }^{15}$

In this case the RCI of 8 points for her chronological age was exceeded for each follow up visit. It was observed that there was a clinically significant change in CISS scores from 44 points to 27 points then finally to 14 points. This indicates that there was a therapeutic change in symptom score between visits hence the dot card orthoptic therapy exhibited therapeutic effect. This is consistent with CITT study group who demonstrated statistically significant change in symptom score for each visit in due course of therapy. ${ }^{3}$ A score of 14 points at the end of therapy indicated success-

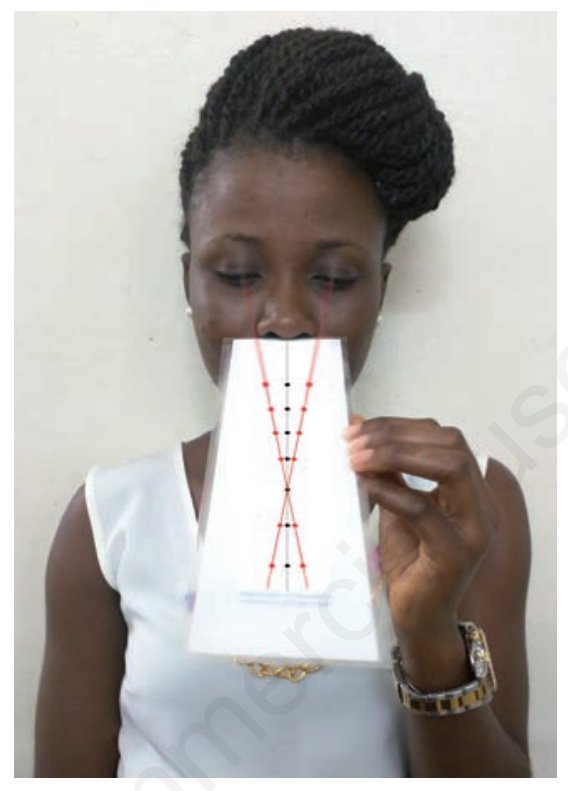

Figure 2. Demonstration of the use of the dot card.

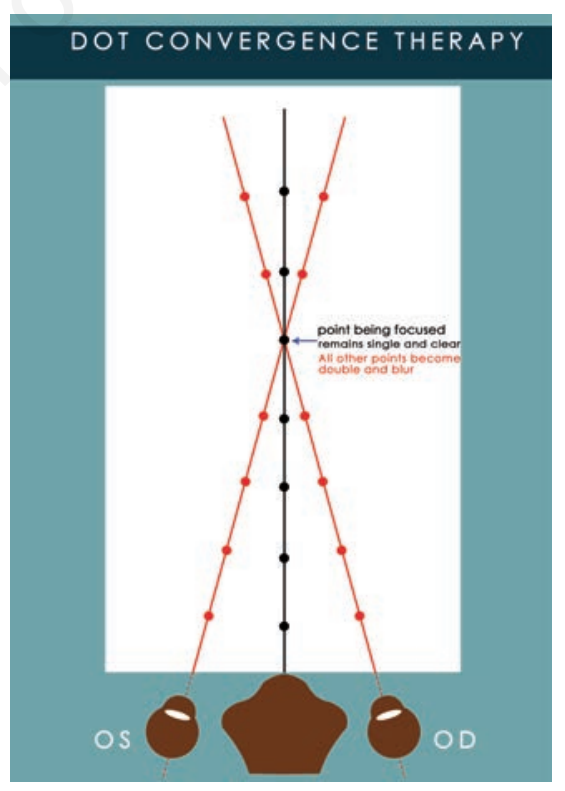

Figure 3. Schema of the dot convergence therapy. ful amelioration of symptoms using the CITT score of less than 16 points. ${ }^{3}$ Using the CITT criteria of $4 \mathrm{~cm}$ as an improvement of NPC, ${ }^{4}$ an NPC changing from $18 / 22$ to $10 / 11 \mathrm{~cm}$ indicated greatly improved NPC. Also positive fusional reserve met Sheard's criteria per the CITT model of using the break point. In this case the demand was 4 prism diopters of exophoria and the positive fusional reserve being 9 prism diopters at the end of last follow-up visit. Also the fact that in this case positive fusional reserve was the same between first and second follow up visits is also consistent with CITT study which reported that most improvement in clinical signs or treatment kinetics for CI patients occur in the first four weeks of thera$\mathrm{py}^{3}$ as was observed in this case for the positive fusional reserve. Hence this patient was successfully treated using the treatment endpoints used by CITT group. ${ }^{3,4}$ Spectacle correction was not prescribed because magnitude of the refractive error was not significant per the criteria by CITT study. ${ }^{4}$

More emphasis was placed on investigating the positive fusional vergence system (rather than the negative fusional system) after first visit because the demand on the binocular system was Exophoria. ${ }^{12}$ The aim was to determine if there had been improvements in the positive fusional reserves to compensate for the Exophoria at near. ${ }^{12}$ The weekly telephone calls to ensure compliance with the home based therapy is consistent with the CITT study. ${ }^{4}$ Successful treatment with vision therapy may suggest that the etiology of CI for this patient was purely innervational in origin as has been indicated by one author. ${ }^{7}$ Maintenance therapy of 15 minutes every week is also consistent with the CITT studies. ${ }^{3,4}$ It is also reported that patients with abnormal vergence system tend to have worse symptoms after thorough binocular vision assessment because these clinical tests tend to stimulate what is the situation when patients are in the real world of near work. ${ }^{16}$ CISS questionnaire has been validated as good measure of acute symptoms of visual discomfort after binocular vision assessment. ${ }^{17}$ In this case our patient demonstrated similar CISS scores of 14 and 13 points before and after binocular vision assessment indicating that her symptoms had been successfully treated. She now compares with normal binocular vision individuals who do not show acute symptoms after binocular vision assessment. This is demonstrated in the CISS scores being similar before and after binocular vision assessment.

\section{Conclusions}

The routine CISS questionnaire administration showed that the patient was very sympto- 
matic leading to a thorough binocular vision assessment. Optometrists should endeavor to perform comprehensive binocular vision test (in addition considering administering validated questionnaires) in order to detect binocular vision anomalies as these conditions are reported to be the most common vision disorders after ametropia. ${ }^{18}$ Also the use of the CISS questionnaire to monitor treatment improvement is recommended to track patient's response to therapy in addition to the clinical signs as demonstrated in this case. The dot card (Figure 1), a simple and easy to perform orthoptic exercise is effective in managing patients with convergence insufficiency hence practitioners especially those in developing countries may use it for vision therapy sessions for their patients. Notwithstanding further research is warranted to investigate the effectiveness of the CISS questionnaire in screening for CI among a larger population of young African patients as a study indicates its ineffectiveness in young university adult populations' elsewhere. ${ }^{19}$

\section{References}

1. Hayes GJ, Cohen BE, Rouse MW, DeLand PN. Normative values for the nearpoint of convergence of elementary schoolchildren. Optom Vis Sci 1998;75:506-12.

2. Borsting EJ, Rouse MW, Mitchell GL, et al. Validity and reliability of the revised convergence insufficiency symptom survey in children aged 9 to 18 years. Optom Vis Sci 2003;80:832-8.

3. Scheiman M, Mitchell GL, Cotter S, et al. A randomized clinical trial of treatments for convergence insufficiency in children. Arch Ophthalmol 2005;123:14-24.

4. Convergence Insufficiency Treatment Trail Study Group. Randomized clinical trial of treatments for symptomatic convergence insufficiency in children. Arch Ophthalmol 2008;126:1336-49.

5. NHS foundation trust. Home exercises to improve convergence insufficiency: patient information. Halton: NHS foundation trust; 2014.

6. Maqsud MA. Orthoptic exercises: a forgotten art. Available from: www.optometry. co.uk/cet

7. von Noorden GK, Campos EC. Binocular vision and ocular motility: theory and management of strabismus. 6th ed. St. Louis: Mosby Inc.; 2002.

8. Burke JP, Shipman TC, Watts MT. Convergence insufficiency in thyroid eye disease. J Pediatr Ophthalmol 1993; 30: 127-9.

9. Schor C. Influence of accommodative and vergence adaptation on binocular motor disorders. Am J Optom Physiol Optics1988;65:464-75.

10. Nayak H, Kersey JP, Oystreck DT, et al. Diplopia following cataract surgery: a review of 150 patients. Eye 2008;22:105764.

11. Cooper J, Pollak GJ, Ciuffreda KJ, et al. Accommodative and vergence findings in ocular myasthenia: a case analysis. J Neuroophthalmol 2000;20:5-11.

12. Scheiman M, Wick B. Clinical management of binocular vision: heterophoric, accommodative, and eye movement disorders. Philadelphia: Lippincott Williams \& Wilkins; 2008.

13. NHS foundation trust. Home exercises to improve convergence insufficiency: patient information. Halton: NHS foundation trust; 2015.

14. Jacobson NS, Truax P. Clinical significance: a statistical approach to defining meaningful change in psychotherapy research. J Consult Clin Psychol 1991;59:12-9.

15. Carmen Barnhardt C, Cotter SA, Mitchell LG, Scheiman M, Kulp MG. Symptoms in children with convergence insufficiency: before and after treatment. Optom Vis Sci 2012;89:1512-20.

16. Cooper J. Clinical implications of vergence adaptation. Optom Vis Sci 1992;69:300-7.

17. Borsting EJ, Rouse MW, Mitchell GL, et al. Validity and reliability of the revised convergence insufficiency symptom survey in children aged 9 to 18 years. Optom Vis Sci 2003;80:832-8.

18. Taub OD, Marc B. Binocular vision anomalies-what every optometrist should know. 2004. Available from: http://www.optometry.co.uk

19. Horwood AM, Toor S, Ridell PM. Screening for convergence insufficiency using CISS is not indicated in young adults. Brit $\mathrm{J}$ Ophthalmol 2014;1:1-5. 